\title{
The Swallowing and Voicing Characteristics of Pharyngeal Dystonia: A Single Case Report
}

\author{
Loren Kahn \\ Heila Jordaan
}

\author{
Department of Speech Pathology and Audiology \\ University of the Witwatersrand \\ Johannesburg \\ South Africa
}

\begin{abstract}
This paper describes the swallowing difficulty and abnormal voicing characteristics of a subject with pharyngeal dystonia. This rare form of dystonia, considered to be a neurological condition resulting in involuntary spasm of the muscles of the pharynx, has not been documented in terms of its effects on the acoustic properties of the voice. This study revealed that during pharyngeal spasm, there are significant delays in voice onset time, a reduction in fundamental frequency, an increased percentage of sub-harmonics and variability in the amplitude perturbation quotient as well as shimmer. There was also evidence of these characteristics during periods of 'spasm-free' voice production, suggesting that the condition might be more consistent than what the subject described. Resonance disturbances were observed in spasm, which might explain the 'hollow' and affected voice quality. The subject also reported severe swallowing difficulties during the periods of spasm, characterised by a tight constriction at the level of the subject's throat. It is clear that an abnormality at the level of the cricopharyngeal muscle has a dual effect on the acoustic properties of the voice and on swallowing.
\end{abstract}

KEY WORDS: pharyngeal dystonia; dysphagia; resonance; acoustic properties; voice onset time

\section{INTRODUCTION}

This study describes the abnormal swallowing and voicing characteristics of a relatively rare condition known as pharyngeal dystonia. The purpose of this research is to contribute to the limited body of literature on this disorder and to raise awareness of the condition among medical and rehabilitation professionals.

According to the : Dystonia Medical Research Foundation (1999), pharyngeal dystonia is a condition in which the muscles of the pharynx contract uncontrollably due to a neurological disruption at the level of the basal ganglia. This may be due to hereditary factors or as a result of trauma, toxins, drugs, neoplasms or infarction. This form of dystonia is one of many dystonias including: involuntary contractions of the eye muscles (blepharospasm), abnormal contractions of the muscles of the head, neck and spine (spasmodic torticollis), muscular rigidity of the jaw, lips and tongue (oromandibular dystonia), muscular spasm of the vocal chords (spasmodic dysphonia) and disturbed fine motor hand functioning (writer's cramp). In mcst patients, the cause of the dystonia cannot be identified. In the early stages of the disorder, dystonia can involve a single muscle group and later progress to others (Aronson, 1985).
No definitive tests exist in the diagnosis of dystonia (Dystonia Medical Research Foundation, 1999): Instead, observations of the abnormal movements, which vary from person to person, are made. This underlines the importance of detailed descriptions of the symptoms of the condition, which enables accurate diagnosis. Current trends in the treatment of dystonia include Botulinum Toxin A (Botox) injections, drug therapy as well as several types of surgical procedures (Moore, 1995). Botox has become the method of choice in treating a variety of dystonias. This substance works by temporarily paralysing the affected muscles and may, in some cases of dystonia, be indicated as the primary form of treatment (Dystonia Medical Research Foundation, 1999).

Only two reports of pharyngeal dystonia could be found in the literature (Kostas Karam, Langhans, \& Vasquez, 1995; Dunne, Hayes, \& Cameron, 1993). Neither of these studies, however, described disordered voicing as part of the symptomatology. In the most recent of these two studies, (Kostas et al., 1995), the researchers described patient's cricopharyngeal dysfunction as being an inadequate relaxation of the cricopharyngeal muscle which kept the larynx from lifting and moving forward. The researchers treated the swallowing disorder using localised injections of Botulinum Toxin A (Botox) into 
the cricopharyngeus muscle. The patient's swallowing was reported to improve significantly following the Botox injections (Kostas et al., 1995).

Prior to this study, the case of an 86 year old male with intermittent mild dysphagia was described, in whom striking recovery occurred in the pharyngeal phase of swallowing following the injection of Botox into the cricopharyngeus muscle (Dunne et al., 1993). As is evident from these two accounts, pharyngeal dystonia is a potentially disabling condition that could be treated successfully if it is correctly identified and diagnosed.

Pharyngeal dystonia is likely to affect both swallowing and voice production because the pharynx comprises the upper end of the respiratory and digestive tract thereby assuming the dual purpose of transmitting air from the nasal cavity to the larynx and food from the oral cavity to the oesophagus

The pharynx is a wide, muscular structure situated behind the nose and mouth and above and around the larynx and consists of striated (voluntary) muscle on the posterior and lateral walls, which enables rapid movements for swallowing and speech. The pharynx consists of the nasopharynx, oropharynx and the laryngopharynx.

The unit functioning of the laryngeal and pharyngeal structures in swallowing and voice production is extremely important when considering a disorder such as pharyngeal dystonia. In cases where swallowing and voice are both affected, one needs to fully understand the anatomical and physiological relationships between the larynx and the pharynx.

The larynx and the pharynx function as a unit, which means that they function jointly during swallowing and voice production.

During the pharyngeal stage of the swallow, for example, the larynx and the hyoid bone are pulled both upward and forward. This movement enlarges the pharynx. It also creates a vacuum in the hypopharynx, pulling the bolus downward (Dobie, 1978; Logemann, 1983, 1989, 1997).

During the laryngeal stage of the swallow, the epiglottis drops down over the top of the larynx, protecting the airway and diverting the bolus into the pyriform sinuses.

The external and internal pharyngeal constrictor muscles play a fundamental part in swallowing (Ekberg \& Nylander, 1981). The external muscles, namely the superior, middle and inferior constrictors, contract in a series of contractile movements from the top down, propelling the food downwards to the oesophagus. The inferior constrictor muscle consists of two parts, a propulsive part known as thyropharyngeus and a sphincteric part known as cricopharyngeus (Groher, 1997). The cricopharyngeus muscle is located in the laryngopharynx, and lies posterior to the cricothyroid cartilage of the larynx (Crafts, 1966). An abnormality at - the level of the cricopharyngeus would cause defective sphincteric operation at the lower level of the pharynx, which may lead to bolus retention in the pharynx (Groher, 1997).

The structures of the larynx are connected to each other and to adjacent structures via ligaments and muscles, within which a state of equilibrium appears to exist (Vilkman, Sonninen, Hurme \& Korkko, 1996). The internal muscles of the pharynx (stylopharyngeus, salpingopharyngeus and palatopharyngeus) and the muscles in the floor of the mouth are involved in the elevation of the larynx during swallowing and speech (Bhatnager \& Andy, 1994 p. 237).

The suprahyoid muscles, i.e., the digastric, the mylohyoid, the geniohyoid, the hyoglossus and the genioglossus muscles as well as the infrahyoid muscles (thyrohyoid, sternothyroid, sternohyoid and omohyoid), have an indirect effect on the larynx which is to move it upward, forward and backward as well as downward in the case of the infrahyoids, (Colton \& Casper,1996). According to Colton and Casper (1996) the lowered laryngeal position results in lengthening of the vocal tract, which has an effect on vocal resonance. These authors also contend that a more direct effect on the voice may result from the restriction of thyroid cartilage movement that is caused by contraction of this muscle group (p. 320). THus affecting vocal pitch regulation through changes in vocal fold length, tension and mass. Other indirect muscular forces include those produced by the palatal, oesophageal, and buchal musculature, of which the palatopharyngeal muscle also has a partial connection to the larynx (Vilkman et al., 1996).

The connection between the larynx and the palatopharyngeus is significant as hyperfunction of the palatopharyngeus muscle is believed to create a 'cul-desac' resonance quality, i.e. a voice quality that is perceived as "hollow and affected." This type of resonance results from a substantial decrease in the dimensions of the oropharyngeal outlet, and is produced when the tongue is retracted into the back of the mouth, causing the origin of the resonance to be situated too posteriorly in the oral cavity (Prater \& Swift, 1984).

The speech production mechanism consists of an air supply, a sound source which sets the air in motion, and a set of resonators that modify the sound in various ways. The lungs supply the air. The sound source is the larynx, where the vocal cords are situated. The filters comprise the organs above the level of the larynx, namely the pharynx, the oral cavity and the nasal cavity. Together, the source and the filters form the vocal tract ( $O^{\prime}$ Grady, Dobrovolsky \& Aronoff, 1993).

Muscular abnormalities of the pharynx, such as Pharyngeal Dystonia will result in 'cul-de-sac' resonance if the involuntary contractions involve more generalised regions of both the upper and lower pharyngeal constrictor muscles. In pharyngeal dystonia, increased muscle contraction could affect the voice in two ways. Firstly, the larynx may be displaced by the spasm, possibly affecting the initiation of voicing, specifically voice onset time. Secondly, the narrowing of the pharyngeal opening due to the abnormal contraction of the posterior pharyngeal wall would alter the physical dimensions of the pharynx, which would affect the resonance properties of the vocal tract.

This study focuses on describing the articulation-laryngeal co-ordination (voice onset time) and acoustic properties of the voice in'order to determine the effect of pharyngeal dystonia on voicing. A brief description of the pharyngeal swallow will supplement the voice data to provide a complete profile of pharyngeal dystonia. 


\section{METHOD}

\section{Aims}

The specific aim of this case report was to describe the swallowing pattern and voicing characteristics of a patient with pharyngeal dystonia.

\section{Subject Description}

The subject of this study approached the Speech and Hearing Clinic of the University of the Witwatersrand for an assessment following a referral from a neurologist. The subject was a 41-year-old English-speaking female. She presented as a subject of convenience and her cooperation was voluntary. Her presence in the clinic provided a unique opportunity to examine the characteristics of her vocal tract in detail. She consented in writing to participate in the study.

The subject (J), presented initially with swallowing difficulties and intermittent periods of "tightness" in voice production. While a neurologist had diagnosed her with pharyngeal dystonia approximately 2 months prior to this study $\mathbf{J}$ reported that she had been experiencing these symptoms for approximately three years.

J's episodes of pharyngeal spasm were initially reported to occur only once or twice a year. However, at the time of this study, she experienced the spasms more regularly (every three to four weeks). $\mathbf{J}$ also reported consistent and marked swallowing difficulties, characterised by a tight throat during swallowing and difficulty getting the bolus down. These swallowing abnormalities were reportedly present all the time and worsened during periods of spasm.

The voicing abnormalities were felt to be present only during periods of spasm and were not as consistent as the swallowing abnormalities. Her subjective reports of spasm were characterised by "tightness" during swallowing and by abnormal voicing, which will be described later in this paper.

J's case history revealed no accompanying trauma, upper respiratory tract infection or neuropsychiatric episode or crisis. The neurological reports ruled extra pyramidal and systemic illnesses. She was not on neuroleptic agents at the time of onset of these symptoms. Furthermore, there was no family history of dystonia. She was treated with the anti-dystonic drugs Artane and Rivotril following her diagnosis two months prior to the study, but no improvement was noted in her condition.

Reports from an ear, nose and throat (ENT) surgeon excluded the presence of any structural abnormality of her larynx. No overt spasms of the adductor vocal apparatus were found. At the time the research was conducted, $\mathrm{J}$ had been assessed by a speech therapist, but she reported that she had not received therapy.

\section{Assessment of the swallow}

A videofluoroscopic examination of the subjects swallow was conducted by a speech pathologist and a radiologist under the supervision of the consulting neurologist. This investigative procedure was conducted at the same time as the voice data was obtained. In this study, videofluoroscopy was used to define the anatomical and physiological abnormalities causing the subject's swallowing difficulties.

\section{Materials}

Standard videofluoroscopic recording equipment was used to record the swallow. The subject was seated on a platform attached to the fluoroscopy table. The swallow was recorded on a videocassette from which, stilled images were later selected using video. editing equipment.

\section{Procedures}

Three consistencies of materials were used in the modified barium swallow to investigate the subject's complaints pertaining to swallowing difficulties. Thin liquid barium (water consistency), barium paste (chocolate pudding mixed with barium paste). and a biscuit coated in barium paste which required chewing. At least two swallows of each material were given to the subject in the following order: $1 \mathrm{ml}, 3 \mathrm{ml}, 5 \mathrm{ml}, 10 \mathrm{ml}$ and cup drinking of thin liquid, 1/3 teaspoon of pudding and $1 / 4$ of an Eat-Sum-More biscuit coated in barium were then given to the subject (Logemann, 1993 p. 169). These substances were given to the subject and she was instructed to feed herself. This was done in an attempt to simulate a normal eating situation. The pharyngeal stage of the swallow was assessed in the antero- posterior view to observe the anatomical symmetry and in lateral view in order to track the bolus on its path down to the oesophagus.

Voice Assessment

\section{Assessment of voice onset time (VOT)}

\section{Equipment}

The subject's voice was recorded in a sound treated booth using a lapel microphone (Ross Multimedia RMA 200) attached $10 \mathrm{~cm}$ from the subject's mouth to maintain a constant mouth-to-microphone distance. Recordings were made using a Sony TCM-359V cassette recorder. Two sets of recordings were obtained on the same day.

\section{Procedures}

The subject was required to produce three consecutive repetitions of the VCV phrases $/ \varepsilon b \varepsilon /, / \varepsilon \mathrm{d} \varepsilon /$ and / $\varepsilon g \varepsilon /$ (Baken, 1987). The first set of recordings were of J's voice in a reportedly 'normal' state while the second set were recorded while $\mathbf{J}$ reported pharyngeal spasm. While EMG recordings would have provided on objective measure of demonstrating spasmodic activity in the pharyngeal muscles, the subject was unwilling to consent to this invasive procedure. It was therefore necessary to rely on the subject's perception of spasmodic activity to determine the presence or absence of pharyngeal spasm.

The absence of spasm was determined by the subject's reports as well as by her ability to swallow normally. During spasm, the subject reported feeling "strangled". Due to the inconsistent nature of the subject's symptoms, it was not possible to set up a time and place to objectively measure her voice using computer software. It was for this reason that audio-taped data was analysed as this did not restrict the researchers and allowed the unpredictable symptoms of this disorder to be recorded. 


\section{Materials}

The specific vowel-consonant-vowel

(VCV) combinations $[\varepsilon b \varepsilon],[\varepsilon \mathrm{d} \varepsilon]$ and $[\varepsilon g \varepsilon]$

were selected for the following reasons: Specific segments of a given utterance are often difficult to identify, thus the use of VCV segments made boundary identification easier (Baken, 1987). The vowel / $\varepsilon /$ was selected for use as there were norms available for its production (Baken, 1987). Plosives were used to measure VOT of segments in the utterances / $/ \mathrm{b} \varepsilon /$, / $\varepsilon \mathrm{d} \varepsilon /$ and / $\varepsilon g \varepsilon /$ as there was a large body of literature documenting norms of production (Baken, 1987).

\section{Data analysis of Voice Onset Time (VOT)}

The Computer Speech Laboratory (CSL) acoustic analysis system was used in the analysis of voice onset time (VOT) (Kay Elemetrics Corporation, 1993). Positive VOT can be defined as the interval in time between the release of a stop consonant and the initiation of glottal pulsing (Colton \& Casper, 1990) and is normally less than $25 \mathrm{~ms}$ for voiced stops (Baken, 1986). In this study, VOT refers to positive VOT as defined above. Time wave displays and narrowband spectrograms were prepared using the CSL software, (Kay Elemetrics Corporation, 1993). The spectrographic displays were used to identify the presence of a stop burst.

VOT measures were made by marking the first evidence of stop release to the onset of voicing. A dual display of the sound wave and a narrowband spectrogram was used. The time cursors of the two displays were linked to ensure more accurate marking. The utterances

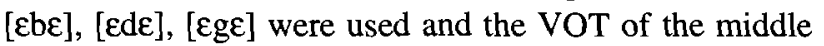
plosives were measured. The time cursor was placed on the moment of plosion ( 0 value). The second cursor was placed at the onset of periodic energy, which is indicative of vocal fold vibration. (Kent $\propto$ Read, 1992). The time lapse between the two cursors was then measured in milliseconds. These values were then compared to the normal value $(25 \mathrm{~ms})$.

\section{Data analysis}

Motion and stilled images of the swallow were analysed to allow the dynamic process of swallowing to be observed in the oral, pharyngeal and oesophageal stages of the swallow.

\section{Acoustic dimensions of voice}

\section{Equipment}

The Multi-Dimensional Voice Program (MDVP) was used to quantitatively assess the subject's voice quality, calculating more than 22 parameters on a single vocalization. The normative references of the MDVP have been based on an extensive database of normal and -disordered voices. Results were graphically and numerically compared to these normative threshold values (Kay Elemetrics Corporation, 1993).

\section{Procedures}

The subject was required to produce a sustained /a:/ for a 5 second duration three consecutive times, which was recorded. Furthermore, it has been suggested that the vowel /a:/ be used to assess the resonating properties of the vocal tract (Prater and Swift, 1984). During the period of spasm experienced by the subject, only two repetitions of /a:/ were obtained due to the variable nature of the presenting spasm which must be considered when interpreting the data.

\section{Data analysis}

In this study, the acoustic parameters that were examined include fundamental frequency, degree of soft harmonics (DSH), amplitude perturbation quotient (APQ) and shimmer. These 5 parameters were selected, as they were the only voice parameters that were consistently found to be abnormal on the Multi Dimensional Voice Programme (MDVP) analysis.

\section{RELIABILITY}

For purposes of reliability, the VOT of the first utterance in each set of utterances (e.g. first [Ebe] of the three) was reanalysed by the researcher, who was blinded to the data labels. The researcher also randomly reanalysed the VOT and Acoustic dimensions of the voice samples.

\section{RESULTS AND DISCUSSION}

\section{SWALLOWING}

The video swallow revealed involuntary muscle contractions of the pharyngeal wall, presented as selected stilled images in Figure 1. Limitations exist in presenting the dynamic process of swallowing using stilled images, as the dynamic sequence of swallowing events is not clearly visible. However, the isolation of individual events during the swallow can be a valuable diagnostic tool, if used in conjunction with a video recording of the event.

The stilled images of the swallow demonstrate the functional abnormalities of the pharyngeal cavity during a single swallow. In the first frame, the subject takes her first mouthful of the liquid barium and achieves adequate oral clearance (frame 2).

In frame 3 , the abnormal muscular contraction of the pharynx is strikingly obvious. In this frame, one can clearly see the barium above the level of constriction, pooling in the valleculae, as well as. barium below the constriction, continuing downwards to the oesophagus. In addition, the pharyngeal walls create a complete constriction, preventing the barium above this level from flowing down towards the oesophagus. This hyperfunction of the pharyngeal walls is described as characteristic of pharyngeal dystonia (Langhans, personal communication 1999).

In the fourth frame, the pharyngeal tube re-opens allowing the barium to flow downwards. At this stage, some degree of abnormality is still apparent at the level of the cricopharyngeal muscle (the fourth cranial vertebra). In the fifth frame, the remaining barium is observed to flow in an anti-gravitational direction back towards the oropharynx, pooling once again in the valleculae. 


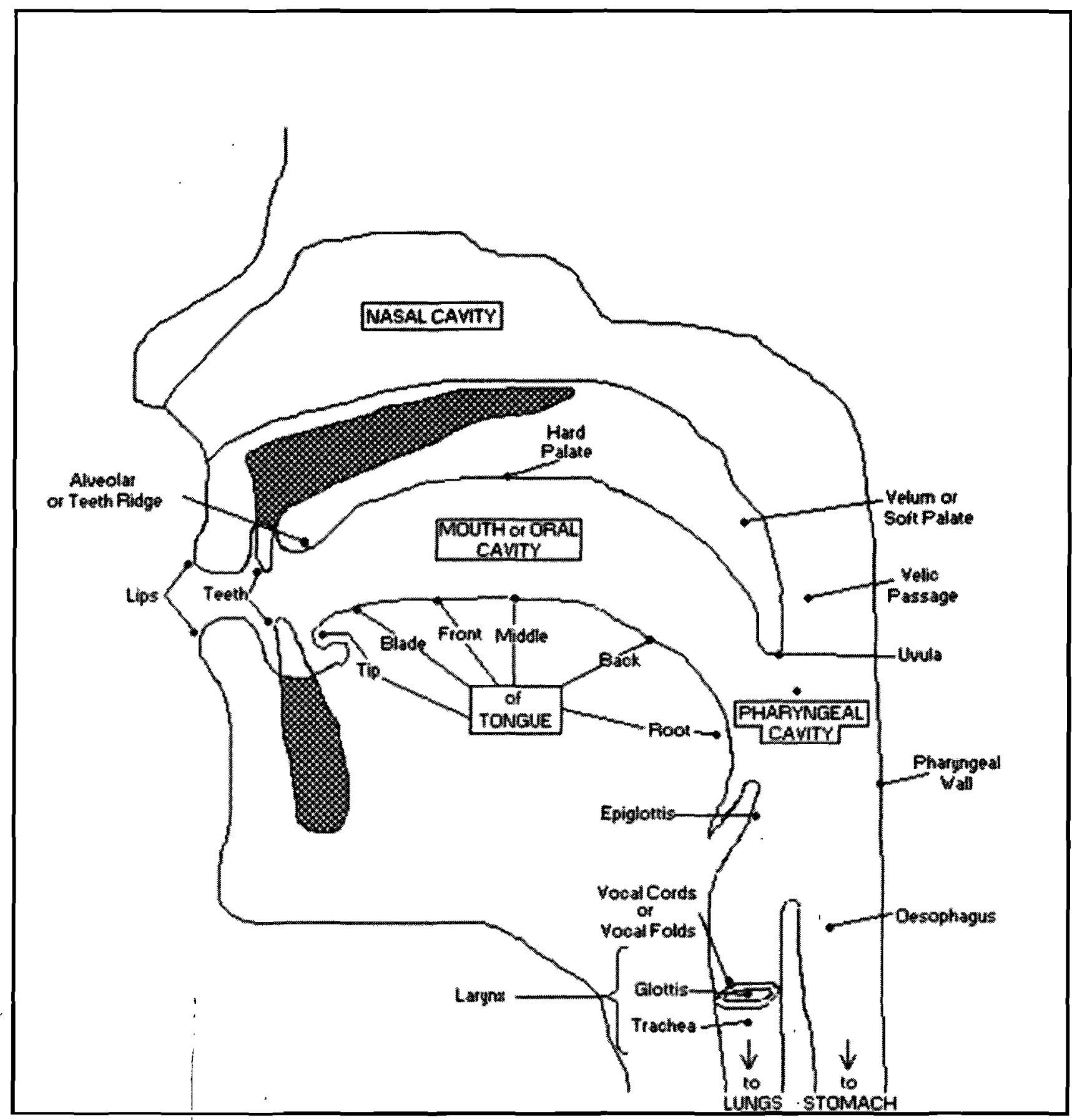

Fig. 1: The anatomical structures of the larynx and pharynx

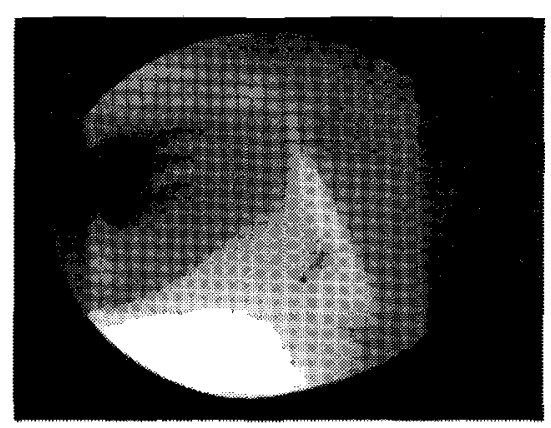

Frame 1

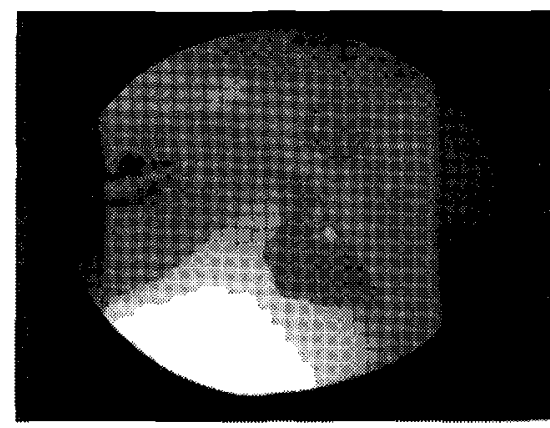

Frame 2 


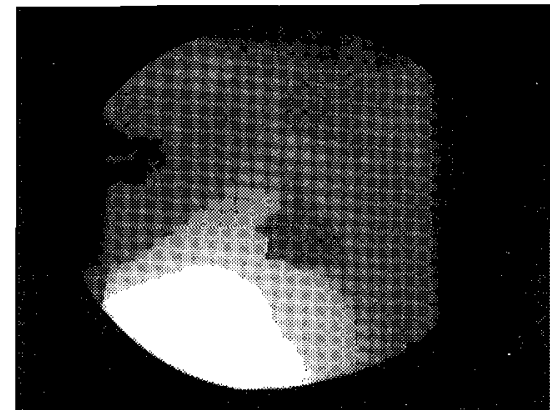

Frame 3

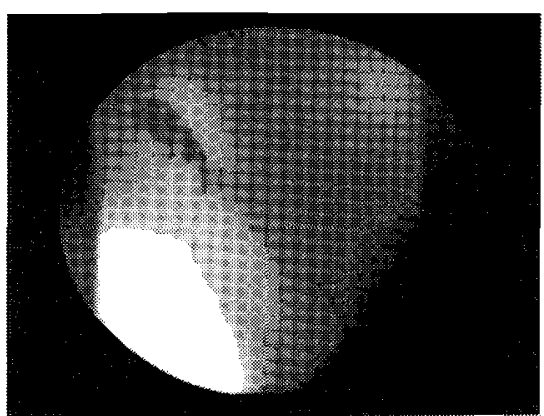

Frame 5

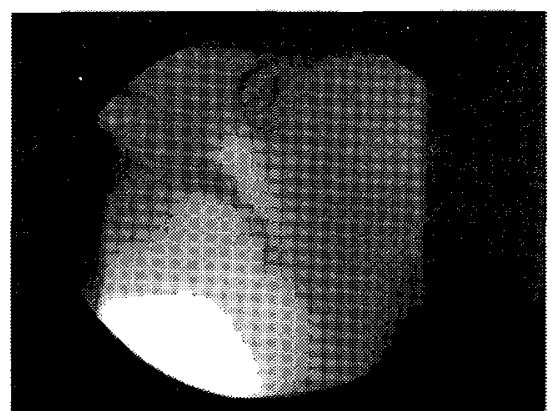

Frame 7

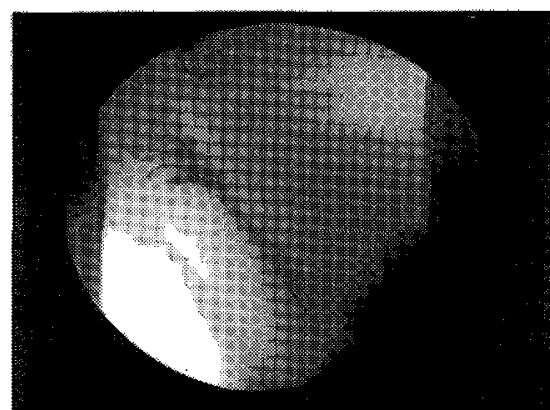

Frame 4

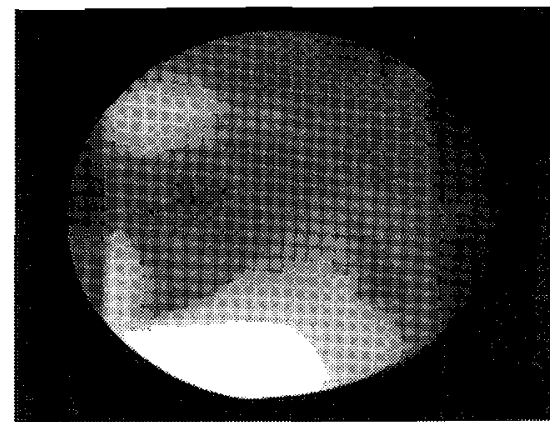

Frame 6

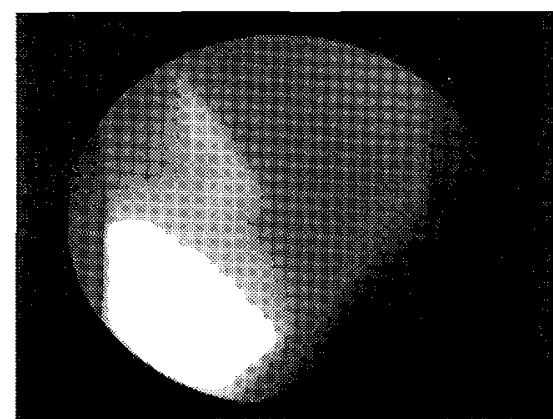

Frame 8

Fig. 1: The abnormal swallowing pattern of a subject with pharyngeal Dystonia

In frame 6, the barium continues upwards, with some evidence of barium in the upper region of the pharynx. In frame 7 , the barium passes up to the base of the tongue, where the pharyngeal phase of the swallow is re-initiated. In the final frame (frame 8), J achieves adequate oral and pharyngeal clearance, and the remaining portion of the barium passes down to the oesophagus for the final phase of the swallow.

\section{RESULTS OF VOICE ONSET TIME MEASURES}

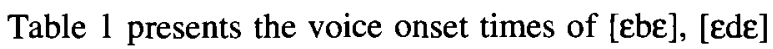
and $[\varepsilon g \varepsilon]$ produced three times each, under two conditions: VOT (1) no spasm, and VOT (2) in spasm.

Figure 2 is a graphic representation of the mean

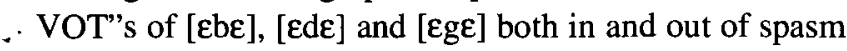
(as per subject's report).

The means and standard deviations for each set of data are provided under both conditions. Reliability measures revealed that re-analysis of the same utterance by the same researcher produced in and out of spasm yielded a value that was within one millisecond of the first measurement. This indicates a high degree of repeatability between measures.
The VOT's recorded during a pharyngeal spasm, were significantly longer in comparison to those recorded during spasm free speech, with an overall mean VOT of $29 \mathrm{~ms}$ during spasm, compared to a mean of $20 \mathrm{~ms}$ without spasm. There were instances of normal voice

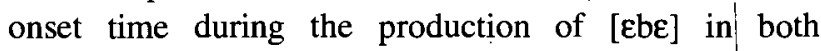
conditions, but the $[\varepsilon \mathrm{d} \varepsilon]$ and $[\varepsilon \mathrm{g} \varepsilon]$ utterances in spasm yielded consistently abnormal results.

Table 1 clearly reflects the delays in VOT during spasm and reflect the subject's inability to co-ordinate her articulators and vocal cords. A muscular spasm within the pharynx disrupts the co-ordinated movement required for normal voicing. Normal VOTs were recorded for production of the bilabial group [Ebe], but the most delayed VOTs were recorded for the $[\varepsilon d \varepsilon]$ group, both with and without spasm.

These findings are significant in light of the fact that the videofluoroscopic study showed the pharynx to be locally affected at the level of the cricopharyngeal muscle. The value of combining videofluoroscopy with acoustic voice measures is clear as the two serve to confirm the isolated observations made using each procedure. 
Table 1: Voice Onset Times for [ $\varepsilon b \varepsilon],[\varepsilon d \varepsilon]$ and $[\varepsilon g \varepsilon]$ in milliseconds (Norm $=25 \mathrm{~ms}$ )

\begin{tabular}{|c|c|c|}
\hline$V C V$ & VOT(1) no spasm (ms) & $\begin{array}{c}\text { VOT(2) in spasm } \\
(\mathrm{ms})\end{array}$ \\
\hline \multirow{6}{*}{$\begin{array}{l}{[\varepsilon b \varepsilon] 1} \\
\text { Reliability check } \\
{[\varepsilon b \varepsilon] 2} \\
{[\varepsilon b \varepsilon] 3} \\
\text { Mean } \\
\text { SD }\end{array}$} & 12.8 & 17.7 \\
\hline & 12.9 & 17.1 \\
\hline & 13.2 & 19.3 \\
\hline & 13.9 & 17.7 \\
\hline & 13.3 & 18.2 \\
\hline & 0.5 & 0.94 \\
\hline \multirow{4}{*}{$\begin{array}{l}{[\varepsilon \mathrm{d} \varepsilon] 1} \\
\text { Reliability check } \\
{[\varepsilon \mathrm{d} \varepsilon] 2} \\
{[\varepsilon \mathrm{d} \varepsilon] 3}\end{array}$} & 22.8 & 41.3 \\
\hline & 22.5 & 42.3 \\
\hline & 28.9 & 36.2 \\
\hline & 29.5 & 38.7 \\
\hline \multirow[t]{2}{*}{ Mean } & 27.1 & 38.7 \\
\hline & 3.8 & 2.74 \\
\hline \multirow{2}{*}{$\begin{array}{l}{[\varepsilon g \varepsilon] 1} \\
\text { Reliability check }\end{array}$} & 16.4 & 29.1 \\
\hline & 16.4 & 31.1 \\
\hline \multirow{3}{*}{\begin{tabular}{l|l}
{$[\varepsilon g \varepsilon] 2$} \\
{$[\varepsilon g \varepsilon] 3$}
\end{tabular}} & 23.4 & 28.4 \\
\hline & 20.7 & 34 \\
\hline & 20.2 & 30.5 \\
\hline & 3.44 & 2.5 \\
\hline
\end{tabular}

The VOT's of the subject's spasm-free voice when producing $[\varepsilon d \varepsilon]$ did not always fall within normal limits (Baken, 1987). This could suggest delayed voice onset time even during spasm free periods, despite the voice being perceived as 'normal' by the researcher and subject during these productions. An important option to consider is that dystonia is characterised by slow, abnormal muscular movements and there may be a short period where the subject is not aware of the spasm. An objective measure of spasm such as electrodes attached to the subject's throat should be used under ideal circumstances to identify instances of unperceived spasm.

\section{RESULTS OF ACOUSTIC MEASURES}

The Multi Dimensional Voice Programme (MDVP) (Kay Elemetrics Corporation, 1993) revealed that some parameters were consistently abnormal during both spasm and spasm free voicing. For the purposes of this case report, only the parameters that fell out of the normal range of values were discussed. 


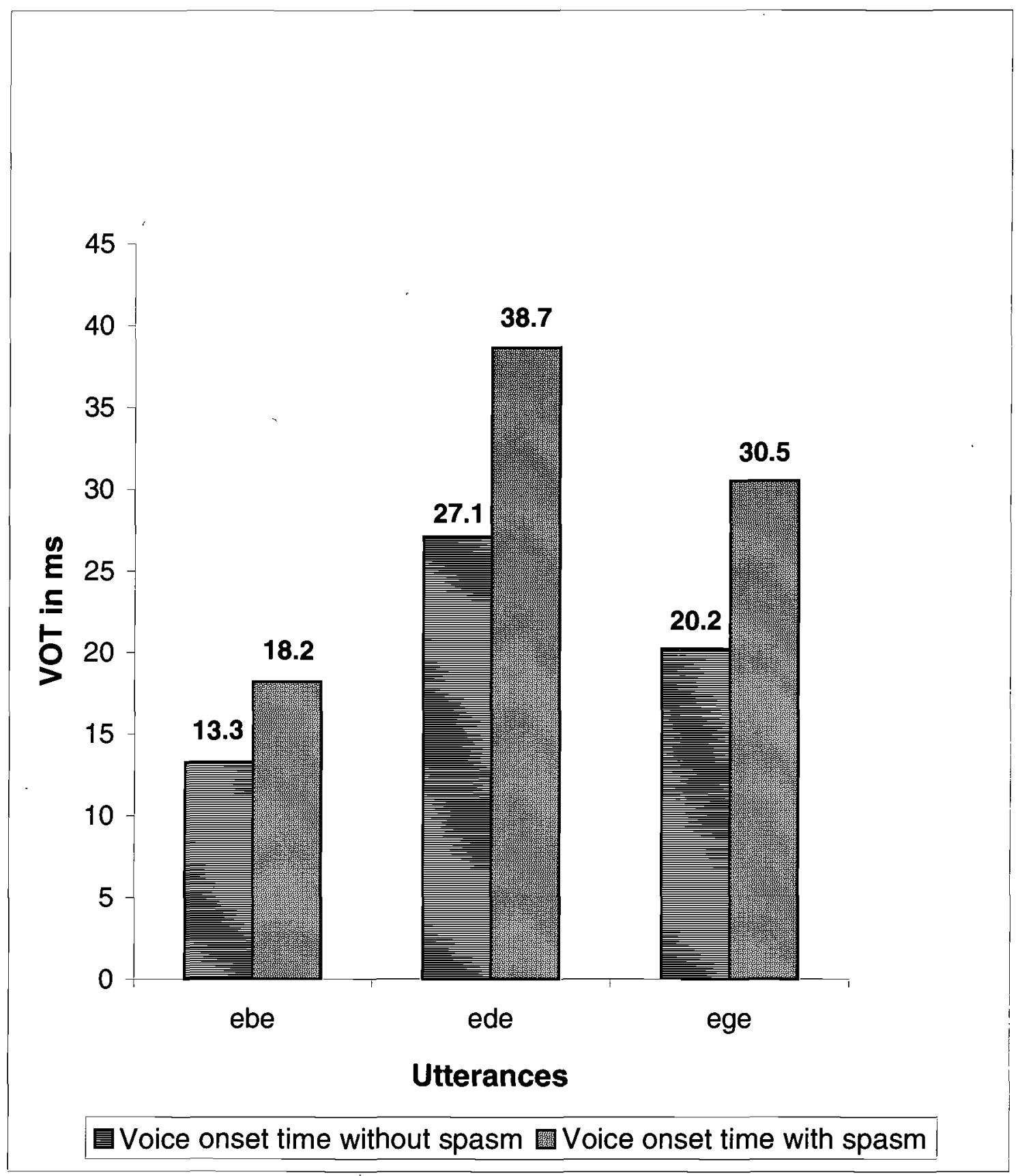

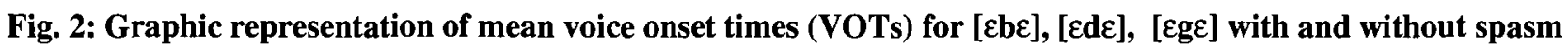

\section{Fundamental Frequency (FO)}

Table 2: Fundamental Frequency (FO) with and without spasm

\begin{tabular}{|l|l|l|l|}
\hline \multicolumn{4}{|c|}{ Fo in Hz } \\
\hline \multicolumn{2}{|c|}{ No spasm } & \multicolumn{2}{c|}{ With Spasm } \\
\hline Initial measure & Repeated measure & Initial measure & Repeated measure \\
& & & 164.10 \\
183.31 & 183.20 & 164.35 & 177.59 \\
.182 .20 & 182.89 & 177.99 & \\
182.51 & 178.81 & & Mean=170.85 \\
Mean=182.67 & Mean=181.63 & Mean=171.17 & \\
\hline
\end{tabular}

Note: FO Norm is $189 \mathrm{~Hz}$. Range is $168 \mathrm{~Hz}-208 \mathrm{~Hz}$.

Table 2 presents the measurements of fundamental frequency (FO) obtained for three productions of the sustained vowel /a:/ during spasm free voice and two productions in spasm. Fundamental frequency reflects the 
vibratory rate of the vocal folds. During spasm free voice, J's FO and thus vibratory rate, consistently fell within normal limits according to normative data (Colton \& Casper, 1990). During spasm, J's FO was lower on

Degree of sub-harmonics (DSH)

Table 3: Degree of Sub harmonics (DSH) with and without spasm average and at times dropped to $164 \mathrm{~Hz}$, falling just below the minimum normal level of $168 \mathrm{~Hz}$ and was not considered to be a significant finding.

\begin{tabular}{|l|l|l|l|}
\hline \multicolumn{5}{|c|}{ DSH (in \%) } \\
\hline \multicolumn{2}{|c|}{ No spasm } & Repeated measure \\
\hline Initial measure & Repeated measure & Initial measure & With Spasm \\
& & & 9.94 \\
5.88 & 16.46 & 5.00 & 8.43 \\
5.75 & 9.20 & 8.24 & \\
Mean $=10.47$ & 4.76 & & Mean $=9.20$ \\
\hline
\end{tabular}

Note: DSH norm $=1.00 \%$

Table 3 represents the measurements of degree of sub-harmonics. DSH is an estimated relative measurement of sub-harmonics to FO components in the voice sample. In the samples that were analysed, there appeared to be variability between the relative amounts of sub-harmonics to FO components. This finding is supported by spectrographic findings, which indicate a visible decrease in harmonics during spasm (when compared to the same segment out of spasm), and out of spasm (when compared to spectrographic analyses of a normal speaker's productions of /a:/). Clear reductions in the degree and amount of harmonics represent the disturbed resonance in J's voice, both during spasm and during spasm free periods. The reduction in harmonics may indicate the presence of aperiodic vocal fold vibration, which is consistent with a spasmodic disorder. Aperiodic vibration may therefore become one of the diagnostic criteria used in the diagnosis of pharyngeal dystonia.

\section{Amplitude Perturbation Quotient (APQ) and Shimmer}

Table 4 indicates the measurements made for the amplitude perturbation quotient (APQ) and shimmer. The APQ evaluates the variability in peak-to-peak amplitude within the voice. Shimmer reflects the cycle-to-cycle variations in amplitude. In spasm free voicing, there was a surprisingly high degree of variability in the amplitude peaks of the voice as compared to the reduced degree of variability observed in spasm. These observations were supported by the reduction in cycle-to-cycle variations of amplitude during spasm. In spasm therefore, reduced variation in peak and cycle amplitude were evident.

Table 4: Amplitude Pertuírbation Quotient (APQ) and Shimmer with and without spasm

\begin{tabular}{|c|c|c|c|}
\hline \multicolumn{4}{|c|}{ APQ (in \%) } \\
\hline \multicolumn{2}{|c|}{ No spasm } & \multicolumn{2}{|c|}{ With Spasm } \\
\hline Initial measure & Repeated measure & Initial measure & Repeated measure \\
\hline 7.17 & 7.00 & 2.98 & 2.83 \\
\hline 3.55 & 4.25 & 3.56 & 3.55 \\
\hline 3.65 & 3.12 & & \\
\hline Mean $=4.73$ & Mean $=4.79$ & Mean $=3.27$ & Mean $=3.19$ \\
\hline \multicolumn{4}{|c|}{ Shimmer (in dB) } \\
\hline Initial measure & Repeated measure & Initial measure & Repeated measure \\
\hline 1.00 & 0.97 & 0.37 & 0.35 \\
\hline 0.46 & 0.56 & 0.42 & 0.42 \\
\hline 0.49 & 0.39 & & \\
\hline Mean $=0.65$ & Mean $=0.64$ & Mean $=0.40$ & Mean $=0.39$ \\
\hline
\end{tabular}

Note: $A P Q$ norm $=3.07 \% \quad$ Shimmer norm $=0.35 \mathrm{~dB}$ 
In all but two instances, the APQ values exceeded the normative value (3.07). This indicates that a high degree of variability exists between the peak-to-peak amplitudes of the analysed voice samples with more variation present in spasm free voice. Using $0.35 \mathrm{~dB}$ as a guideline for the presence of shimmer according to normative data (Baken 1987), it appeared that there was generally more shimmer present in spasm free voice when compared to the voice sample with spasm.

The overall reduction in the APQ and in the degree of shimmer may be due to the increased tension of the vocal cords during spasm. This finding may assist in the diagnosis of pharyngeal dystonia as a decrease in the APQ value and reduced shimmer may indicate an overall reduction in the amplitude of the voice.

\section{Summary of Voice Measures}

Generally, J's voice contained some identifiable deviations from what would be considered normal, both during pharyngeal spasm and during periods of spasm free voicing. This may suggest that there is a degree of sub clinical acoustic irregularity in this subject's voice despite the apparent lack of awareness or perceptual evidence of such abnormality.

\section{CONCLUSIONS}

This research was a first attempt at describing the swallowing and voice characteristics of pharyngeal dystonia. It is significant that the dysphagia was so easily identifiable and that so many abnormal laryngeal and acoustic parameters were observed in the voice of this subject. This study could thus be seen as a preliminary guide for the development of a future diagnostic protocol in cases where pharyngeal spasm may be suspected.

Comprehensive follow up of this case obviously needs to be implemented to determine whether the voice symptoms in particular, become more consistent and identifiable over time.

The findings of this study confirm the importance of the pharynx with regards to its resonance properties as well as its effect on the structures of the larynx during times of pharyngeal spasm. The findings in this case indicate that the pharynx is of considerably more importance in voice production than most textbooks have acknowledged. The need for more research into the specific relationship between the larynx and the pharynx will make the disorder of pharyngeal dystonia easier to understand.

The integrated functioning of the pharyngeal structures is emphasised in the literature (Greene, 1972). This study suggests that both gross and fine movements of the pharynx have the potential to drastically alter the resonance characteristics of the voice. Resonance disturbances are clearly observable when comparing the - degree of sub harmonics in the sustained production of the /a:/ vowel produced in spasm to the same vowel produced with normal resonance. The resonance disturbance in the subject's voice could be described as cul-de-sac resonance that can be observed using spectrographic data.

The subject's self perceptions of 'tight' voice production have physiological reality. Evidence of these abnormalities exists in the physiological and acoustic abnormalities observed during periods of spasm in this study. In addition, the acoustic results of this study show clear secondary effects on the larynx. Despite the absence of reported or perceived laryngeal abnormalities in the case being studied, secondary effects are caused by the pharyngeal muscle spasm resulting in voice disturbances, such as the significant delay in voice onset times during spasm.

This study bears implications for a treatment programme for $\mathrm{J}$ and provides a baseline measurement from which the success of any intervention can be evaluated. At this stage, treatment options for this case are limited due to the lack of available resources and the inconsistent nature of her symptoms. Botox has been used in the treatment of some cases of pharyngeal dystonia (Kostas et al., 1995; Dunne et al., 1993). Botox injections into the cricopharyngeal muscle would result in a paralysis of the pharynx.

In South Africa, Botox has become increasingly popular in the treatment of a variety of dystonias. Few specialists, both in South Africa and internationally, are experienced in placing the injection because the cricopharyngeal muscle is hidden behind the cricoid cartilage. Inaccurate injection could paralyse other muscles in the area, resulting in a worse dysphagia. A paralysed pharynx could benefit $J$ during periods of spasm, but due to the inconsistent nature of her symptoms these injections could be to her detriment during periods of no spasm.

The fact that very few cases of pharyngeal dystonia have been documented does not mean that they do not exist, instead, it suggests that perhaps too little is known about the signs and symptoms of this disorder which may affect it's diagnosis. The need for further research into this disorder is essential so that clinicians diagnose and manage individuals with pharyngeal dystonia more accurately.

\section{REFERENCES}

Aronson, A. E. (1985). Clinical Voice Disorders - An Interdisciplinary Approach ( $2^{\text {nd }}$ Edition.). New York: Thieme.

Baken, R. J. (1987). Clinical Measurement of Speech and Voice. London: Taylor Francis Ltd.

Bhatnager, S. C. \& Andy, O. J. (1994). Neuroscience For The Study of Communicative Disorders. Baltimore: Williams and Wilkins.

Colton, R. H. \& Casper, J. K. (1990). Understanding Voice Problems - a Physiological Perspective for Diagnosis and Treatment. Baltimore: Williams and Wilkins.

Crafts, R. C. (1966). A Textbook of Human Anatomy. Roland Press: New York.

Dobie, R. (1978). Rehabilitation of Swallowing Disorders. American Family Physician, 17, 84-95.

Dunne, J., Hayes, M. \& Cameron, D. (1993). Botulinum A toxin for cricopharyngeál dystonia. Lancet, 6, 342-559.

Dystonia Medical Research Foundation (1999). Understanding Dystonia. Chicago: Illinois.

Ekberg, O. \& Nylander, Ǵ. (1981). Pharyngeal constrictor paresis in patients with dysphagia: a cineradiographic study. Clinical Radiology, 33, 253. 
Greene, M. C. L. (1972). The Voice and Its Disorders. Great Britain: Pitman Press.

Groher, M. E. (1997). Dysphagia- Diagnosis and Management (3rd ed.). Massachusetts: ButterworthHeinemann.

Kay Elemetrics Corporation. (1993). MultiDimensional Voice Program (MDVP) Model 4305 Operations Manual: New Jersey.

Kent, J. F. \& Read, C. (1992). The Acoustic Analysis of Speech. San Diego: Singular Publishing Group, Inc.

Kostas, S. P., Karam, F., Langhans, J. L. \& Vasquez, A. B. (1995). Treatment of Dysphagia Resulting From Cricopharyngeal Dysfunction with Botox: Preliminary Thoughts and Observations. The Florida Journal of Communication Disorders, 15 , 22-25.

Logemann, J. (1983). Evaluation and Treatment of Swallowing Disorders. Austin, Texas: Pro-Ed.

Logemann, J. (1989). Short Course in Diagnosis and Remediation of Swallowing Disorders. Oakland, California.
Logemann, J. A. (1993). Manual for the videofluoroscopic study of swallowing. Austin. Texas. Pro-Ed.

Logemann, J. (1997). Structural and Functional Aspects of Normal and Disordered Swallowing. In Ferrand and Bloom (Eds.), Introduction to Organic and Neurogenic Disorders of Communication (pp. 229. 246). Boston: Alyn and Bacon Publishers.

Logemann, J. A. (1998). Evaluation and Treatment of Swallowing Disorders - 2nd ed.). Texas: Pro-Ed.

Moore, A.P. (1995). Handbook of Botulinum Toxin Treatment. Oxford: Blackwell Science.

O'Grady, W., Dobrovolsky, M. \& Aronoff, M. (1993). Contemporary Linguistics: An Introduction (2nd ed.). USA: Martins Publishers.

Prater, R. J. \& Swift, R. W. (1984). Manual of Voice Therapy Little, USA: Brown and Company.

Vilkman, E., Sonninen, A., Hurme, P. \& Korkko, P. (1996). External Laryngeal Frame Function in Voice Production Revisited: A Review. Journal of Voice, 10(1), 78-92. 